\title{
Evaluation of Electrochemical and Anticorrosion Properties of Polyaniline-Fly Ash Nanocomposite
}

\author{
Karthika Shetty $\mathbb{D},{ }^{1}$ Jayadev $\mathbb{D},{ }^{2}$ Kalyan Raj $\mathbb{D},{ }^{3}$ and H. C. Ananda Murthy $\mathbb{D}^{4}$ \\ ${ }^{1}$ Department of Chemistry, RNS Institute of Technology, Visvesvaraya Technological University, India \\ ${ }^{2}$ Department of Chemistry, SJB Institute of Technology, Visvesvaraya Technological University, India \\ ${ }^{3}$ Department of Chemistry, BMS College of Engineering, Visvesvaraya Technological University, India \\ ${ }^{4}$ Department of Applied Chemistry, School of Applied Natural Science, Adama Science and Technology University, Adama, \\ P.O. Box 1888, Ethiopia
}

Correspondence should be addressed to Karthika Shetty; karthikashetty09@gmail.com and H. C. Ananda Murthy; anandkps350@gmail.com

Received 18 October 2021; Revised 28 November 2021; Accepted 30 November 2021; Published 20 December 2021

Academic Editor: Michael J. Schütze

Copyright (c) 2021 Karthika Shetty et al. This is an open access article distributed under the Creative Commons Attribution License, which permits unrestricted use, distribution, and reproduction in any medium, provided the original work is properly cited.

\begin{abstract}
In India, the thermal station generates approximately $6.9 \times 10^{7}$ tons of fly ash (FA) as a waste by-product. As part of this work, little attempt was made to produce useful materials from waste material. In our current research, polyaniline- (PANI-) fly ash (FA) nanocomposite (PFNC) was synthesized using an in situ polymerization method. The synthesized composites were characterized by employing advanced analytical, microscopic, and spectroscopic tools. The results of the X-ray diffraction (XRD) analysis confirm the effective reinforcement of FA into PANI in PFNC. The presence of functional groups in PFNC has been confirmed by Raman and FT-IR spectroscopic techniques. The SEM micrographs of the nanocomposite revealed the presence of agglomerated and fragmented structures in PFNC. The weight loss for PFNC was observed to occur in three stages as revealed by thermogravimetric analysis (TGA). UV-visible spectra for PFNC proved that FA stabilized the PANI in emeraldine form. Electrodynamic polarization studies were conducted to explore the corrosion resistance of nanocomposite-coated mild steel. The corrosion current density $\left(i_{\text {corr }}\right)$ for PFNC-coated mild steel (MS) specimens was found to decrease when compared to the bare substrate, indicating superior corrosion resistance in PFNC-coated substrate. Similarly, Tafel and cyclic polarization studies too confirmed superior anticorrosion property for MS coated with PFNC.
\end{abstract}

\section{Introduction}

Sustaining and enhancing environmental quality have become the major challenge of the current situation [1]. Increased environmental pollution affects life system on earth. Composites based on PANI with metal oxides, have been reported in the literature. Fly ash is the waste product generated from power plants. Most fly ash contains silica, alumina, ferric oxide, and calcium oxide [3]. Depending on the composition of the coal, the mineralogical properties of the fly ash vary, so the composition of the pollutant also varies. Different disposal methods were needed depending on the composition of the coal; thus, the disposal method is expensive [4].
FA is an electrically insulating compound. Adding fly ash into the PANI matrix regulates the conductivity of material [5]. The fly ash has high electrical resistivity [4]. The high electrical resistivity, low thermal conductivity, and low density of fly ash may be helpful in making lightweight insulating composites. In the recent years, fly ash has rapidly gained momentum due to its low cost and ecological source [6]. The current use of fly ash depends solely on its microstructure and chemical composition.

PANI continues to be of interest because the amine nitrogen atoms can be oxidized and protonated to modify its electrical and optical characteristics. PANI is recognized for its good thermal and environmental durability, but its insolubility and fragility [4] make it difficult to treat, limiting 


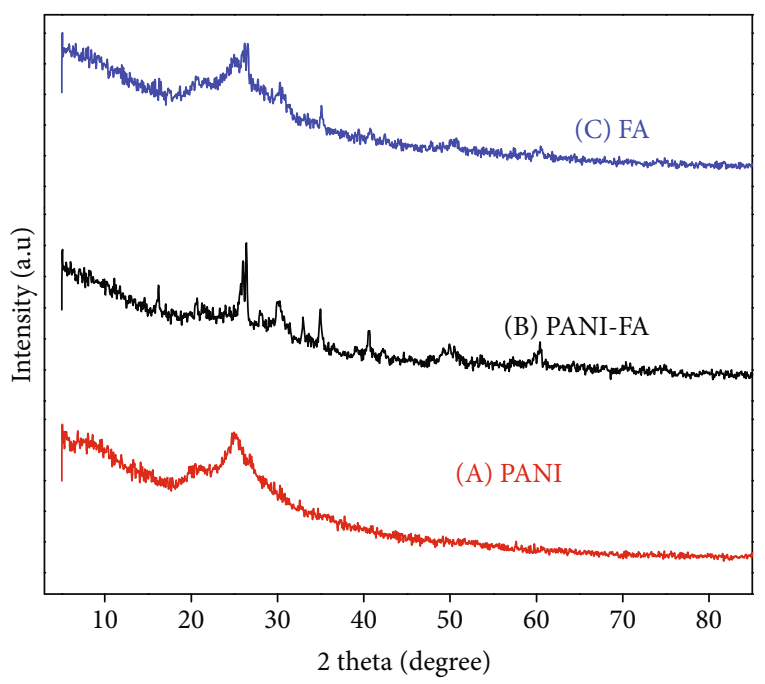

Figure 1: PXRD patterns of (a) PANI, (b) PANI-FA (PFNC), and (c) FA.

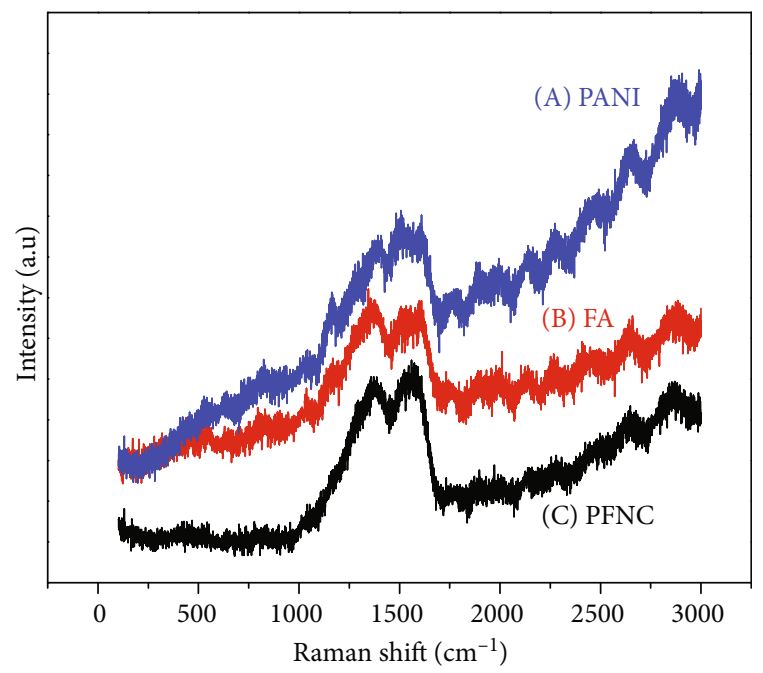

Figure 2: Raman spectra of (a) PANI, (b) FA, and (c) PFNC.

its commercial applicability. PANI is highly stable; it is a cost-effective conducting polymer. It is used as a photosensitizer; it is used in rechargeable battery, anticoating display system [7]. Conductive polymers had been used because of their unique qualities such as mechanical strength, electrical conductivity [5], corrosion resistance, stability, and capacity to be synthesized of both oxidative and electrochemically [4]. As a result, PANI is useful in a wide range of applications including solar power conversion, rechargeable batteries, electrochromic displays, electrochemical sensors, capacitors, and active corrosion protector. Due to the ease of synthesis, processing environmental stability, and low synthetic cost, polyaniline is probably the most important industrial conducting polymer today. PANI can be synthesized electrochemically or chemically by using an appropriate oxidizer in an acid medium. PANI is available in three forms: completely oxidized (prenigraniline), completely

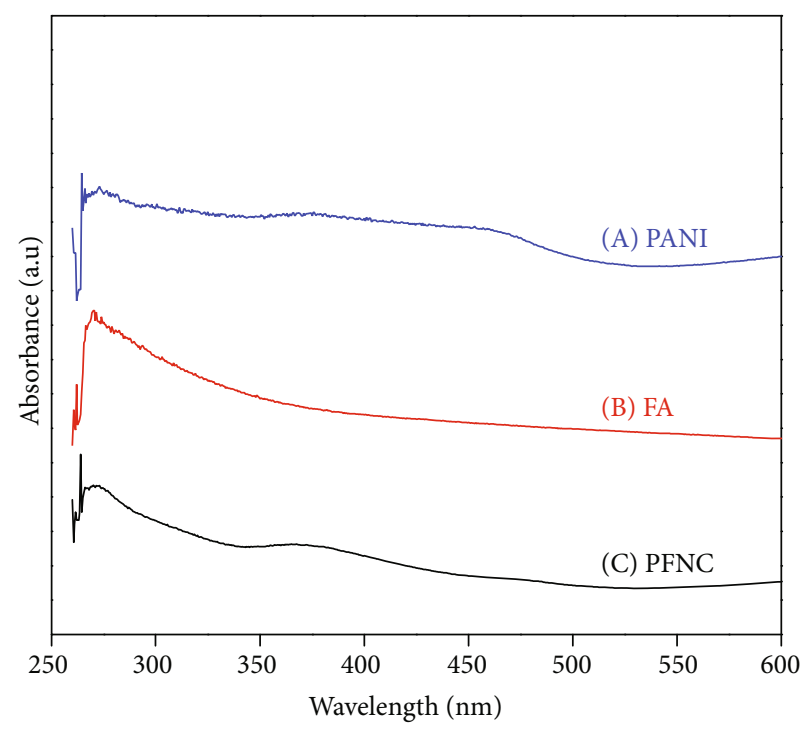

FIgure 3: UV-visible spectra of (a) PANI, (b) FA, and (c) PFNC.

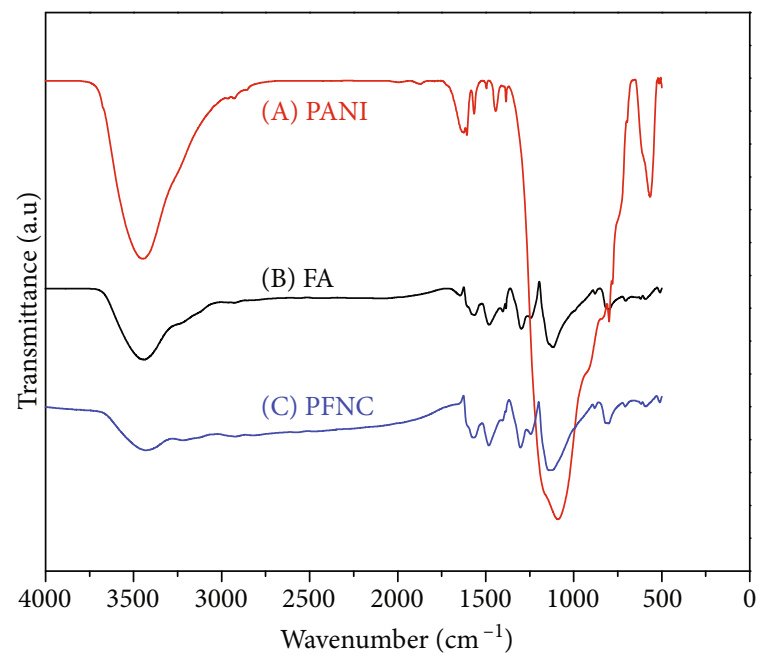

Figure 4: FTIR spectra of (a) PFNC, (b) FA, and (c) PAFC.

reduced (base of leucoemeraldine), and half oxidized (emeraldine base). Semioxidized form is a more stable form of PANI, and it is conductive when it is subjected to doping [4]. It effectively gives another approach for modifying PANI's conductivity to obtain desired result in the context of a potential application. Furthermore, because the processes are underlying the variation of conductivity of PANI and other conducting polymers are yet unknown, a study of these materials is likely to shed light on this topic as well. In India, the thermal station generates approximately $6.9 \times 10^{7}$ tons of fly ash as a waste by-product. In this work, we have aimed to synthesize a nanocomposite by using fly ash as reinforcement for PANI matrix to get PFNC. The synthesized composites were subjected to characterization using several techniques. Finally, the electrochemical corrosion characteristics were investigated for the synthesized nanocomposite PFNC by Tafel and cyclic polarization methods. 


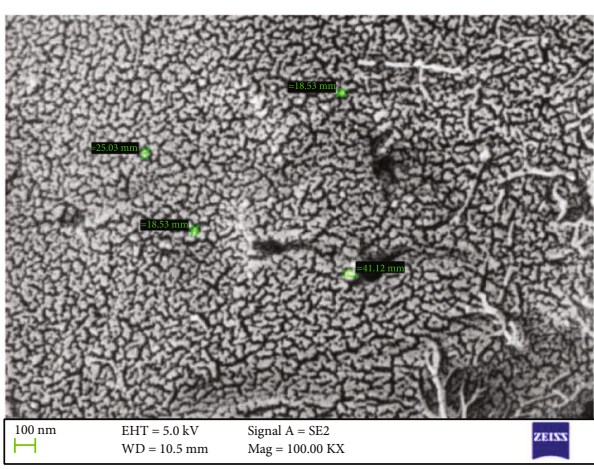

(a)

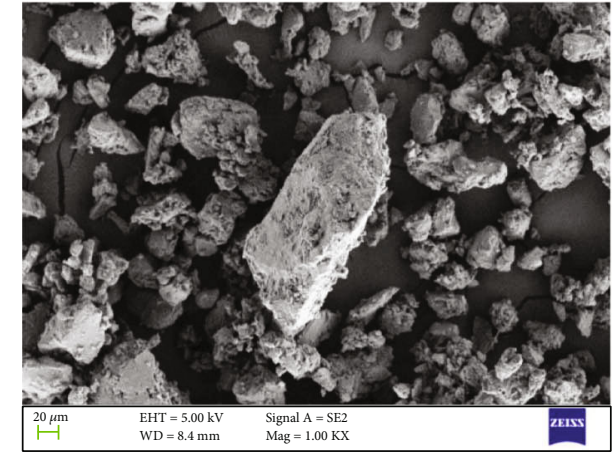

(b)

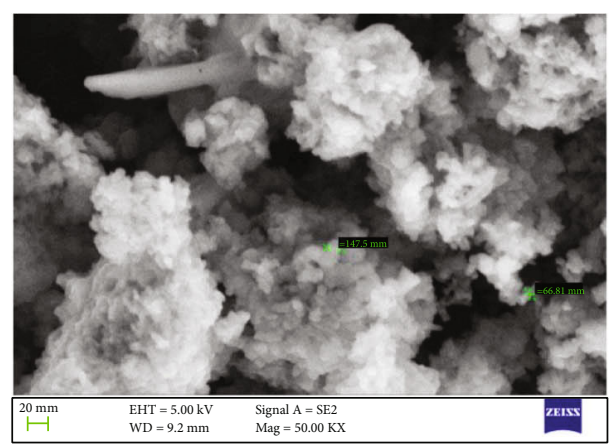

(c)

Figure 5: The SEM images of (a) PANI, (b) FA, and (c) PFNC.

\section{Materials and Methods}

2.1. Materials. Chemicals aniline, ammonium persulphate (APS), and $\mathrm{HCl}$ (AR grade) were purchased from SD FineChem Ltd. Double distilled aniline was used in PANI synthesis. Fly ash sample was collected from thermal plant Raichur. The distilled aniline was used in this work.

2.2. Polyaniline Synthesis. In situ polymerization was used to make the PFNC nanocomposite. Here is a typical preparation method: in situ polymerization was used to make the distilled aniline. The distilled aniline $(5 \mathrm{~mL})$ was dropped into a $70 \mathrm{~mL}$ beaker containing $2 \mathrm{M} \mathrm{HCl}$ [7] under sonication. The calculated amount of APS was dissolved in a known quantity of demineralized water; then, it is added to the above mixture with vigorous stirring for 20 minutes. The mixture was kept for 10 to $12 \mathrm{hrs}$ with constant stirring. The reaction mixture was filtered with $2 \mathrm{M} \mathrm{HCl}$ solution and demineralized water, followed by acetone. Then, it is dried in an electric oven for $14 \mathrm{hrs}$. The obtained product is pure PANI [8].

2.3. Synthesis of PFNC. The in situ polymerization mode was adapted to synthesize PFNC nanocomposite. $1 \mathrm{~g}$ of FA was taken in a beaker containing $70 \mathrm{~mL}$ of $2 \mathrm{M} \mathrm{HCl}$ solution and stirred for $10 \mathrm{~min} .5 \mathrm{~mL}$ of aniline was added to the solution with vigorous stirring, and the mixture was kept for $12 \mathrm{hrs}$ to complete reaction. Then, $5 \mathrm{~g}$ of APS was dissolved in demineralized water, mixed to the above solution within 20 min of time interval. The reaction mixture was kept for stirring for $12 \mathrm{hrs}$ at room temperature. Later, this mixture was allowed to settle overnight, and it is filtered, with $2 \mathrm{M}$ $\mathrm{HCl}$, followed by demineralized water and acetone. The final product obtained was a faint green colour precipitate of PFNC.

2.4. Characterization. X-ray powder diffraction (PXRD) measurements were done with a Bruker D8 ADVANCE ECO with scan range $10-800$ using $1 \mathrm{KW} \mathrm{Cu}-\mathrm{K} \alpha(\lambda=$ 1.54A0). TGA measurements were carried out with the Shimadzu DTG-60 instrument. About $10 \mathrm{mg}$ of sample was taken in an alumina pan at $10^{\circ} \mathrm{C}$ per minute heating rate in the range of $20-900^{\circ} \mathrm{C}$ under a nitrogen atmosphere. Fourier transform infrared spectroscopy (FTIR) measurement was performed using Bruker TENSOR II of wave number $500-4500 \mathrm{~cm}^{-1}$. UV-visible spectroscopy measurement was performed by using Shimadzu MPC360000 in the range of 200 to $900 \mathrm{~nm}$. Raman absorption measurement was performed by the LabRam HR spectrometer in the region of $100-1800 \mathrm{~cm}^{-1}$. Surface morphology of nanocomposite was studied by the SEM Hitachi SU 3500 instrument. Corrosion studies were carried out using an electrochemical workstation, CHI608E (USA). Platinum electrode as indicator electrode, $\mathrm{Ag} / \mathrm{AgCl}$ acting as reference electrode, and mild steel as working electrode were utilized during the electrochemical studies.

\section{Results and Discussion}

3.1. PX-Ray Diffraction Studies. The diffraction pattern of PANI (Figure 1(a)) displayed a broad signal intensity peak at $25.4^{\circ}$ which is due to emeraldine salt along with numerous 


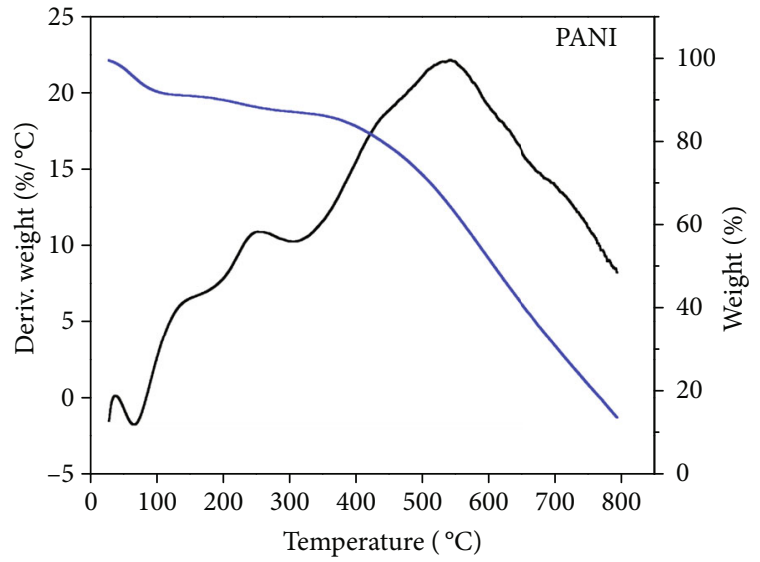

(a)

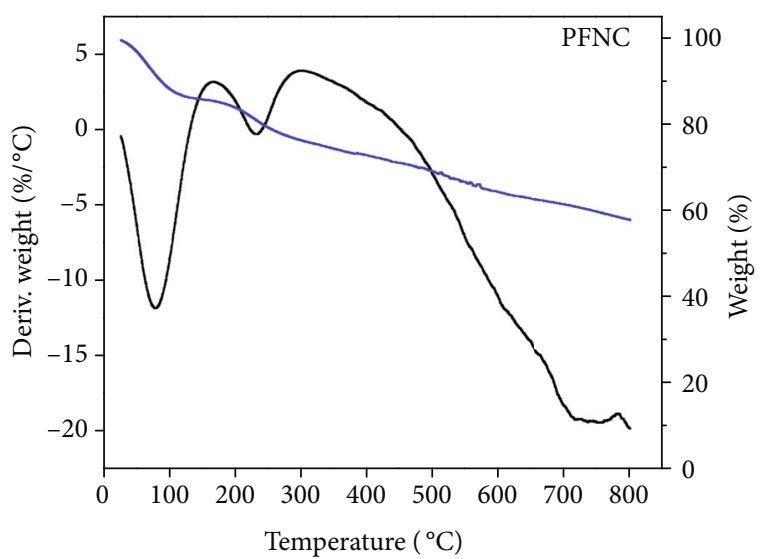

(c)

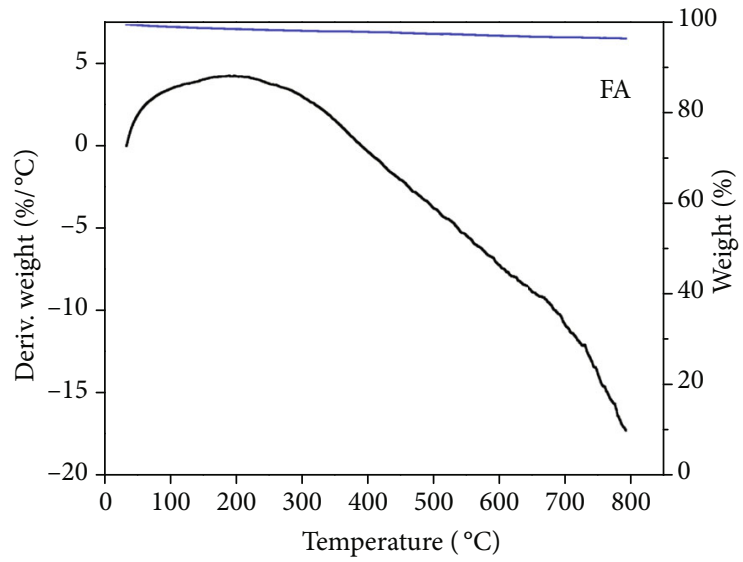

(b)

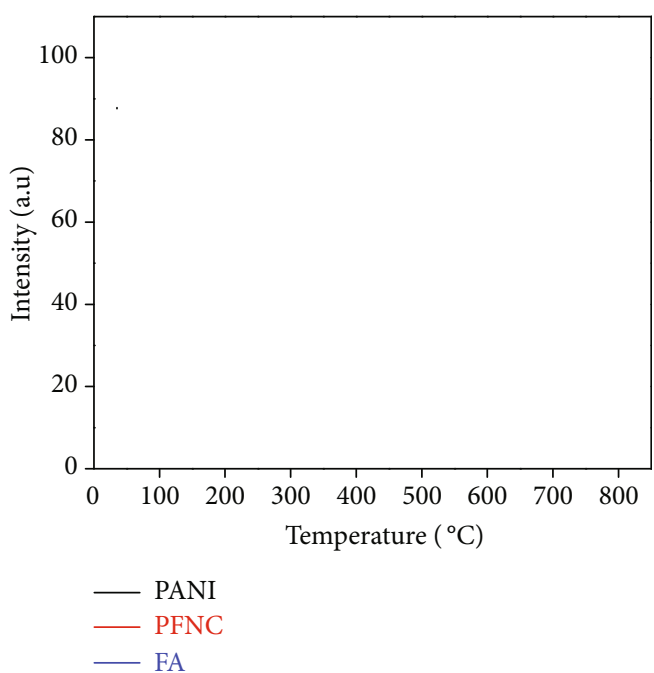

(d)

Figure 6: TGA curves of (a) PANI, (b) FA, (c) PFNC, and (d) compiled plot for PANI-FA-PFNC.

low signal intensity peaks [4]. FA contains crystalline quartz and mullite, as well as a little amount of glassy phase with a signal intensity of $25.01^{\circ}$ (Figure $1(\mathrm{c})$ ). Signal strength is as shown in Figure 1 (b) for PFNC at $26.84^{\circ}$ as reported by Narayan et al. [9]. In PFNC, the amorphous structure of PANI has been transformed to a semicrystalline state. Since FA consists of three major components of crystalline phases of alumina, silica, and mullite and along with amorphous phases of these materials, it is obvious that PANI loses its shape as a result of interfacial interaction with fly ash. PFNC composite peak resembles all of PANI and FA characteristic peaks. The results of the XRD confirm the effective reinforcement of FA into PANI and also that the composite acts like semicrystalline without any structural changes [6].

In PFNC nanocomposite, FA shows the diffraction peak at the most stable surface. This dominates the crystalline structure of the doped nanocomposite. Scherrer's formula (equation (1)) was used to deduce the crystallite size:

$$
d=\frac{0.9 \lambda}{\beta \cos \theta},
$$

where $\lambda$ is the wavelength of $\mathrm{X}$-ray, $d$ is the particle size in diameter, $\beta$ is the FWHM of the surface plane pattern [10], and $\theta$ is the diffraction angle. The average mean crystallite size was deduced to be $15 \mathrm{~nm}$.

3.2. Raman Spectroscopy. Figure 2 depicts the Raman spectra of PANI, FA, and PFNC. Figure 2(a) shows multiple peaks for PANI at $438 \mathrm{~cm}^{-1}$ due to phenazine [11], peak at $574 \mathrm{~cm}^{-1}$ due to $\mathrm{C}-\mathrm{H}$ of the benzenoid ring [11], peak at $1103 \mathrm{~cm}^{-1}$ due to $\mathrm{C}-\mathrm{H}$ of the quinoid ring, and peak at $1378 \mathrm{~cm}^{-1}$ for semiquinone rings. In case of FA (Figure 2(b)), the peak at $1348 \mathrm{~cm}^{-1}$ can be attributed to $\mathrm{C}-\mathrm{N}$ stretching, peaks at $1240 \mathrm{~cm}^{-1}$ are due to $\mathrm{C}-\mathrm{H}$ bending, peak at $1593 \mathrm{~cm}^{-1}$ is due to $\mathrm{C}=\mathrm{C}$, and peak at 1760 corresponds to $\mathrm{C}=\mathrm{O}$ stretching. $\mathrm{PFNC}$ retains almost all the peaks of PANI and fly ash corroborating the formation of nanocomposite (Figure 2(c)). The presence of some peaks near $1000 \mathrm{~cm}^{-1}$ is due to interaction of molecular oxygen and carbon.

3.3. UV-Visible Spectroscopy. UV-visible spectra for PFNC composite prepared in DMF solution are presented in Figure 3. The spectra show absorbance peaks at $274 \mathrm{~nm}$ 
TABLe 1: The data obtained during TGA analysis of PANI, FA, and PFNC.

\begin{tabular}{|c|c|c|c|c|c|}
\hline \multirow{2}{*}{ PFNC $(w / w \%)$} & \multirow{2}{*}{ Process } & \multicolumn{3}{|c|}{ Transition temperature range $\pm 2^{\circ} \mathrm{C}$} & \multirow{2}{*}{ Weight loss (\%) } \\
\hline & & $T_{i}$ & $T_{\max }$ & $T_{c}$ & \\
\hline \multirow{4}{*}{ PANI } & 1 & 26 & 132 & 197 & 10 \\
\hline & 2 & 197 & 250 & 324 & 3 \\
\hline & 3 & 324 & 544 & 799 & 74 \\
\hline & Leftover & & & & 13 \\
\hline \multirow{4}{*}{ PFNC } & 1 & 23 & 156 & 227 & 25 \\
\hline & 2 & 227 & 294 & 723 & 30 \\
\hline & 3 & 723 & 780 & 799 & 5 \\
\hline & Leftover & & & & 40 \\
\hline \multirow{2}{*}{ FA } & 1 & 24 & 217 & 799 & 3 \\
\hline & Leftover & & & & 97 \\
\hline
\end{tabular}

TABle 2: Tafel parameters for PFNC in $0.1 \mathrm{M} \mathrm{HCl}$ solution.

\begin{tabular}{lccccc}
\hline Sp.no & $i_{\text {corr }}\left(\mathrm{Acm}^{2}\right)(\mathrm{A})$. & $E_{\text {corr }}(\mathrm{v})$ & CR $(\mathrm{mpy})$ & Beta-a $(\mathrm{mV} / \mathrm{dec})$ & 3.112 \\
\hline PANI & $1.262 \times 10^{-3}$ & -0.0512 & $4.177 \times 10^{2}$ & 2.962 & 4.626 \\
FA & $1.766 \times 10^{-3}$ & -0.0612 & $3.612 \times 10^{2}$ & 2.162 & 3.116 \\
$25 \mathrm{ppm}$ & $2.617 \times 10^{-3}$ & -0.0711 & $3.112 \times 10^{2}$ & 1.917 & 3.132 \\
$50 \mathrm{ppm}$ & $3.166 \times 10^{-3}$ & -0.0827 & $2.962 \times 10^{2}$ & 1.776 & 2.962 \\
$75 \mathrm{ppm}$ & $0.916 \times 10^{-3}$ & -0.0810 & $1.916 \times 10^{2}$ & 1.612 & 3.113 \\
$100 \mathrm{ppm}$ & $1.116 \times 10^{-3}$ & -0.0913 & $1.766 \times 10^{2}$ & & \\
\hline
\end{tabular}

and $363 \mathrm{~nm}$ due to pi-pi transition of the benzenoid and pipi quinoid ring of PANI. For FA, a small peak was observed at $259 \mathrm{~nm}$ and no peaks were found in the wavelength range between 600 and $700 \mathrm{~nm}$. PFNC composite resembles all the characteristic peaks of PANI and FA. In the case of composite, the absence of a peak at $274 \mathrm{~nm}$ indicates stabilization of emeraldine form of PANI. By comparing PANI peak with that of composite, spectra prove that FA stabilized the PANI in emeraldine form.

3.4. FT-IR Spectroscopic Studies. The presence of PANI in the fly ash matrix can be seen in the FTIR peaks as shown in Figure 4. Peaks for PANI occurred around $3449 \mathrm{~cm}^{-1}$, $1578 \mathrm{~cm}^{-1}, 1483 \mathrm{~cm}^{-1}, 1373 \mathrm{~cm}^{-1}, 1273 \mathrm{~cm}^{-1}, 1021 \mathrm{~cm}^{-1}$, and $587 \mathrm{~cm}^{-1}$ corresponding to $\mathrm{N}-\mathrm{H}$ stretching, $\mathrm{C}=\mathrm{N}$ stretching [12], quinoid stretching, benzenoid ring stretching, C-N stretching, $\mathrm{C}-\mathrm{H}$ in-plane bending, and $\mathrm{C}-\mathrm{H}$ out-of-plane bending [11], respectively (Figure 4(a)). In raw fly ash, as shown in Figure 4(b), the presence of broad peaks at $3400 \mathrm{~cm}^{-1}$ and $1600 \mathrm{~cm}^{-1}$ was attributed to the stretching and deformation vibrations of $\mathrm{OH}$ and $\mathrm{H}-\mathrm{O}-\mathrm{H}$ groups from the water molecules. The peaks for FA at $1087 \mathrm{~cm}^{-1}$ and $559 \mathrm{~cm}^{-1}$ correspond to in-plane and out-of-plane $\mathrm{C}-\mathrm{H}$ bending vibrations. The $\mathrm{Si}-\mathrm{O}-\mathrm{Si}$ and $\mathrm{Fe}-\mathrm{O}$ stretchings caused peaks at $620 \mathrm{~cm}^{-1}$ and $1094 \mathrm{~cm}^{-1}$, respectively. The PFNC composite maintains nearly all of the PANI and FA peaks (Figure 4(c)).

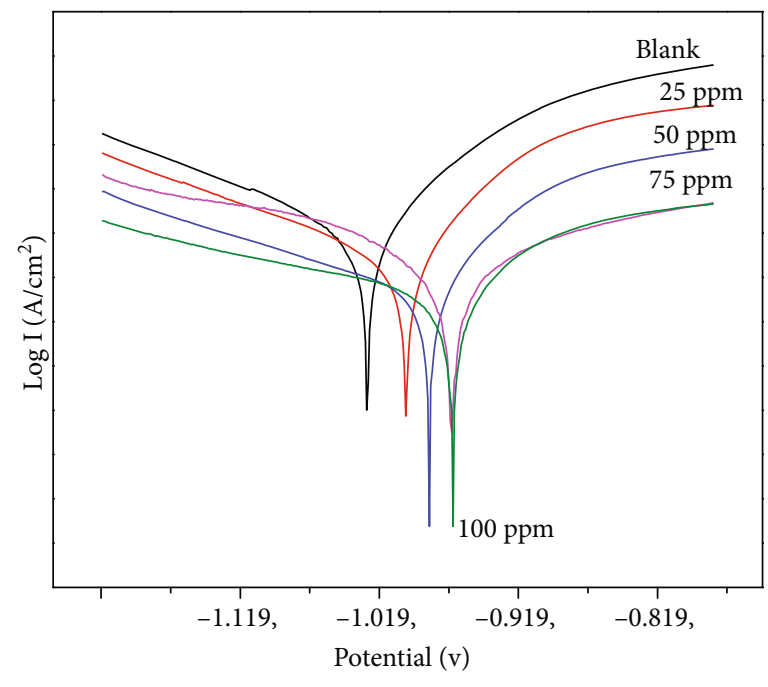

Figure 7: Tafel plots of PANI reinforced by FA.

3.5. Morphological Studies of PANI, FA, and PFNC. Figure 5 depicts the SEM images of PANI, FA, and PFNC. The image of FA consists of hollow spheres with regular smooth texture along with some glassy unshaped fragments [13] as shown in Figure 5(b). Homogeneous dispersion of FA particles was observed at high magnification. The SEM image of PANI shows porous and nonuniformly agglomerated structures (Figure 5(a)). The micrographs of PFNC composite reveal 


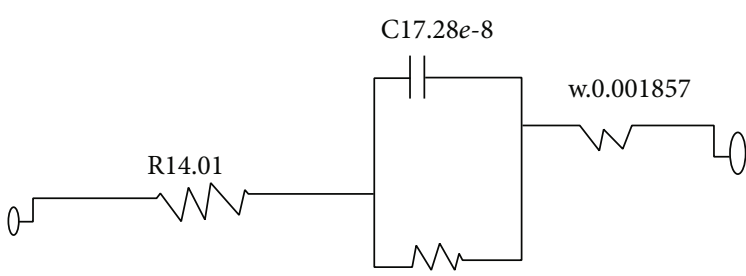

(a)

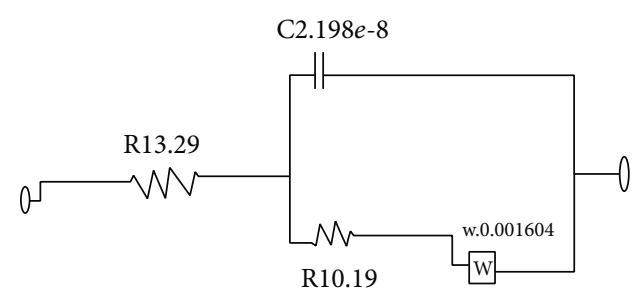

(b)

Figure 8: EIS circuits of (a) bare and (b) epoxy-coated mild steel.

that the agglomeration and fragment structure of polyaniline is preserved even after addition of FA [14]. As a result, we conclude that the matrix of FA and fragments of PANI were uniformly dispersed in PFNC nanocomposite (Figure 5(c)).

3.6. Thermogravimetric Analysis. The weight loss of a substance as a function of temperature is measured using a TGA study. The TGA curves are shown in Figure 6. The TGA plot for PANI is presented in Figure 6(a) which shows 3 weight loss ranges. Similarly, the TGA plot for FA is presented in Figure 6(b) with a gradual weight loss. The weight loss for PFNC was observed to occur in three stages. In the temperature range of 23 and $227^{\circ} \mathrm{C}$, the first stage of weight loss occurred, with a $20 \%$ moisture loss. The second weight loss occurred in the range of $227-723^{\circ} \mathrm{C}(28 \%)$ is attributable to the degradation of aliphatic structure and other thermally unstable FA molecules [15]. The breakdown of the leftover polymer backbone causes the third stage of weight loss at about $799^{\circ} \mathrm{C}(52 \%)$ as presented in Figure 6(c). The cumulative TGA plots for PANI, FA, and PFNC are presented in Figure $6(\mathrm{~d})$. The data obtained during TGA analysis is presented in Table 1.

3.7. Corrosion Studies. Step 1. Mild steel (MS) substrate was cut into a $2 \mathrm{~cm} \times 2 \mathrm{~cm}$ square plate. To remove rust, the substrate is cleaned with dilute sulphuric acid and then sonicated to eliminate contaminants. After that, it was rinsed in acetone and dried with a drier.

Step 2. Corrosion investigations are conducted on a precleaned steel substrate. A fixed volume of Araldite epoxy resin is combined with various PFNC compositions having thickness of $0.289 \mathrm{~mm}$. These produced compositions are mechanically coated on MS substrates and dried in an oven at $60^{\circ} \mathrm{C}$ for $60 \mathrm{~h}$. Then, utilizing an electrochemical workstation and an EIS, corrosion studies were conducted.

3.7.1. Potentiodynamic Polarization (Tafel) Studies. The electrochemical measurements were carried out utilizing a three-electrode system that included a PFNC-coated MS substrate as the working electrode, an $\mathrm{Ag}$-AgCl electrode as the reference electrode, and a platinum electrode as the working electrode.

The results of potentiodynamic polarization of samples coated with FA-doped and unreinforced epoxy layers in $0.1 \mathrm{HCl}$ solutions [16] are presented in Table 2. Figure 7 shows the Tafel plots produced after 48 hours of immersion in $0.1 \mathrm{HCl}$ solution for the bare substrate and epoxy-coated substrate. The corrosion current density $\left(i_{\text {corr }}\right)$ for coated specimens was found to decrease by about one order of magnitude as compared to the bare substrate, indicating superior corrosion resistance in coated substrate [17]. The shift in the $E_{\text {corr }}$ value to a greater positive value for the PFNC-coated substrate to the bare substrate indicates that the surface is being protected [17].

In comparison to epoxy coatings with $75 \mathrm{ppm}$ and $100 \mathrm{ppm}$ (loading of PANI-FA), the $i_{\text {corr }}$ values of epoxy coatings with $75 \mathrm{ppm}$ and $100 \mathrm{ppm}$ (loading of PANI-FA) are two orders of magnitude and more than two orders of magnitude (Table 2). Furthermore, the presence of significantly greater anodic and cathodic Tafel constants for specimens $75 \mathrm{ppm}$ and $100 \mathrm{ppm}$ suggests that PFNC has a significant role in influencing anodic and cathodic corrosion reactions. The considerable drop in $i_{\text {corr }}$ values for specimens $75 \mathrm{ppm}$ and $100 \mathrm{ppm}$ are due to the strong corrosion protection provided by PFNC, which is included as an ingredient in epoxy resin. The epoxy resin's composite functions as a physical barrier against corrosion, protecting the underlying metal surface. Furthermore, in an acidic solution, FA particles provide mechanical integrity to the coating. In this way, it works similarly to other conventional coatings that prevent ions from passing through. The PANI, which provides anodic protection to the steel surface by moving its potential to the passive region, is the main difference between conventional coatings and coatings with PANI-FA composite. This is due to conjugated polymers' high oxidative properties, which act as an oxidant on the steel surface. PFNC is used as an additive in the epoxy resin in this case. However, the role of PANI in intercepting and transporting electrons at the metal surface cannot be ruled out, and it improves the corrosion resistance of coatings. As a result, PFNC prevents mild steel surface from corroding. It strengthens the epoxy coating's corrosion resistance in this way. However, as more PFNC was loaded into epoxy, the lesser is the $i_{\text {corr }}$ value. When compared to epoxy coatings, the observed $i_{\text {corr }}$ values for specimens $25 \mathrm{ppm}$ and $50 \mathrm{ppm}$ are nearly four times greater (Table 2). This could be attributed to the negative effects of PFNC levels below $50 \mathrm{ppm}$ in epoxy systems. The optimum limit of PFNC loading in epoxy is thought to be beyond $50 \mathrm{ppm}$.

3.7.2. Nyquist Polarization Studies. MS substrate was exposed to $0.1 \mathrm{HCl}$, and impedance was measured and analyzed using appropriate equivalent electric circuits for bare and coated MS substrates [18]. To fit the experimental impedance data, several equivalent electric circuits were analyzed and tested. After fitting, the circuit with the least error 

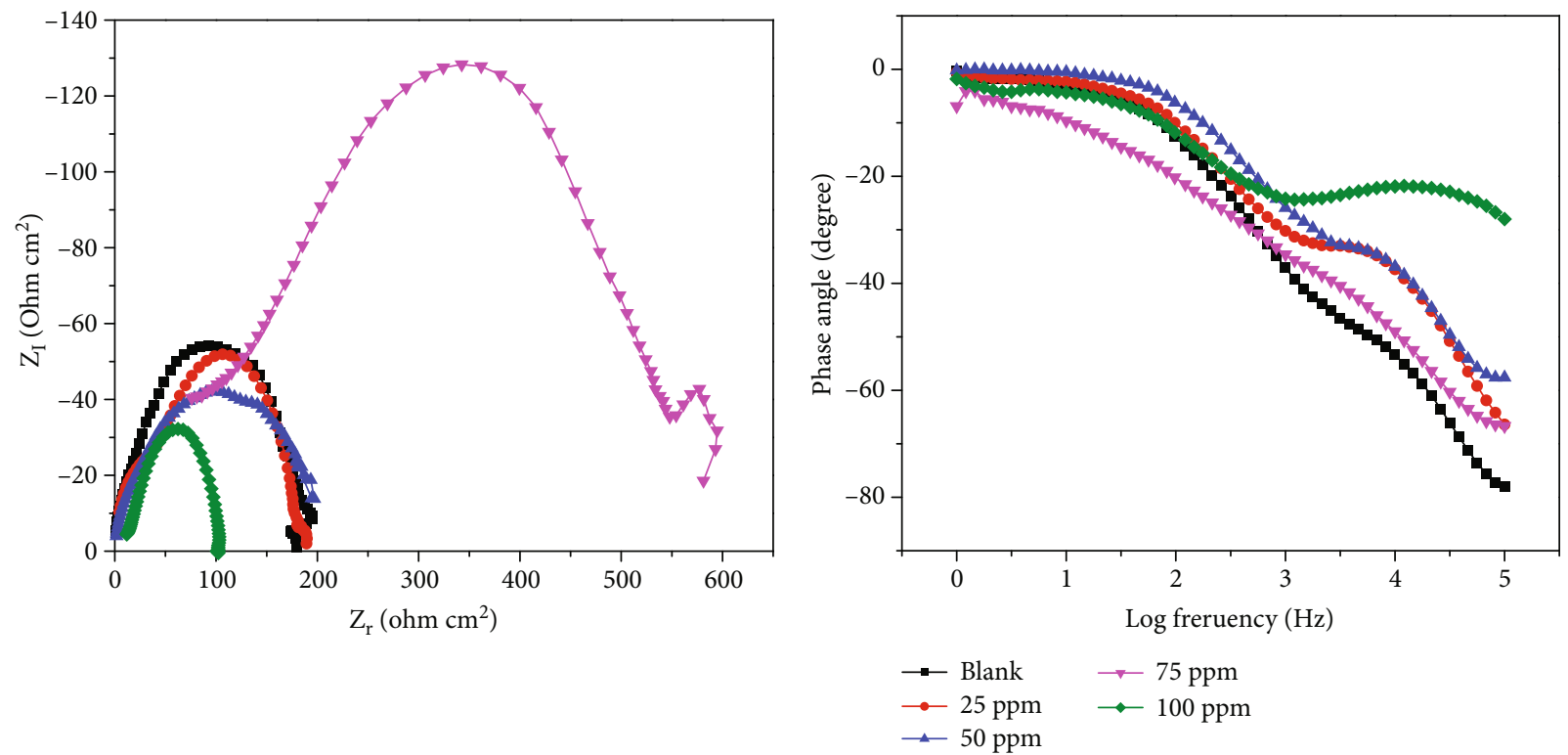

(a)

(b)

Figure 9: Nyquist plot for (a) bare and (b) epoxy-coated mild steel in acidic condition.

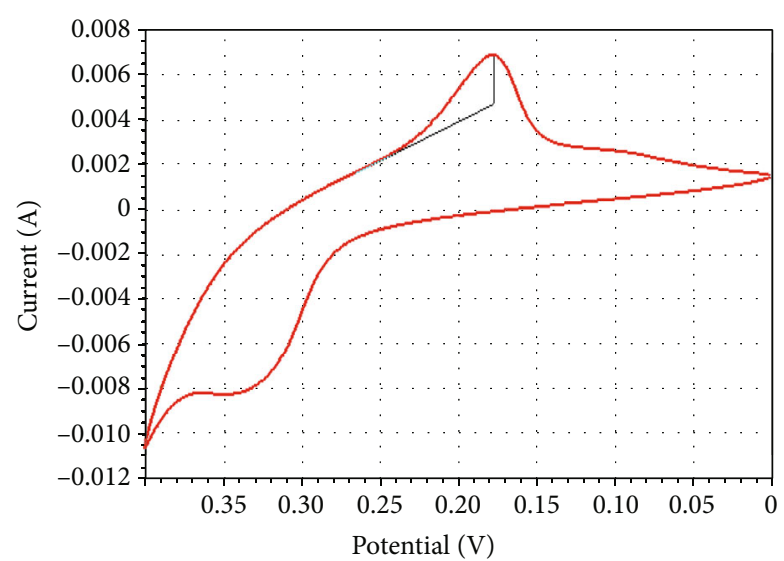

(a)

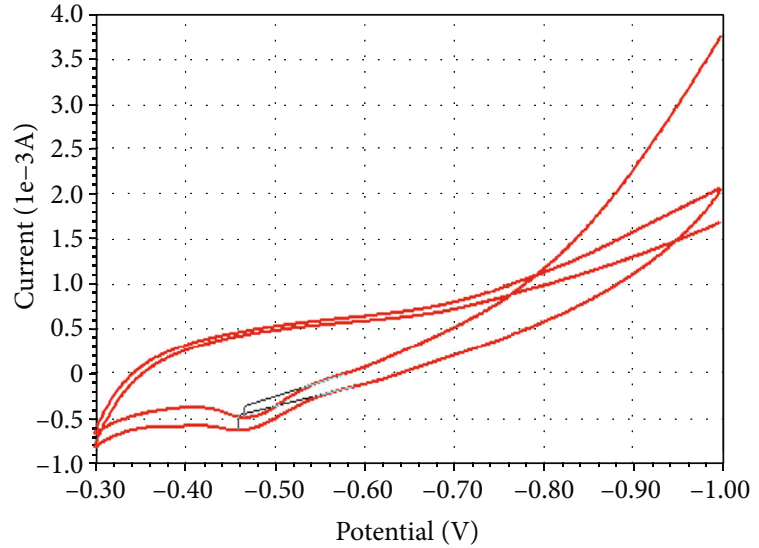

(b)

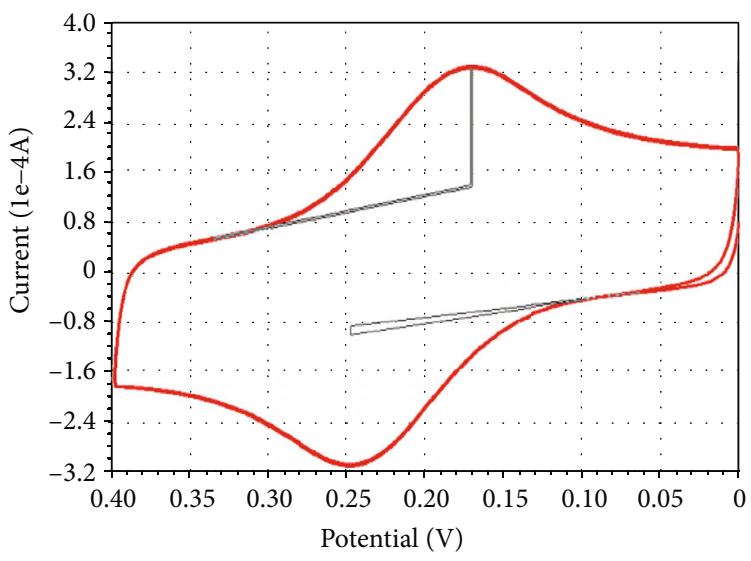

(c)

Figure 10: Cyclic voltammetry of (a) $25 \mathrm{ppm}$, (b) $50 \mathrm{ppm}$, and (c) $100 \mathrm{ppm}$. 


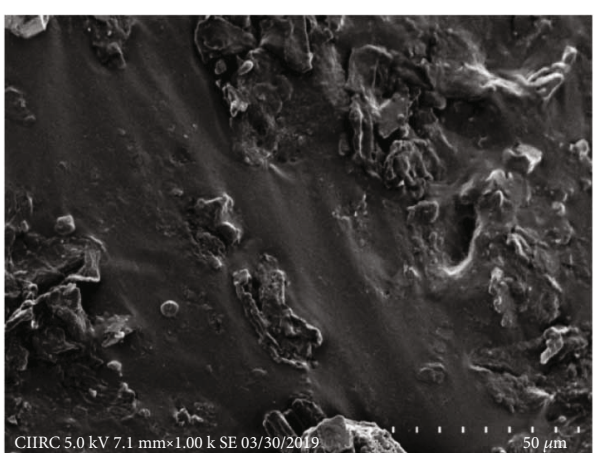

(a)

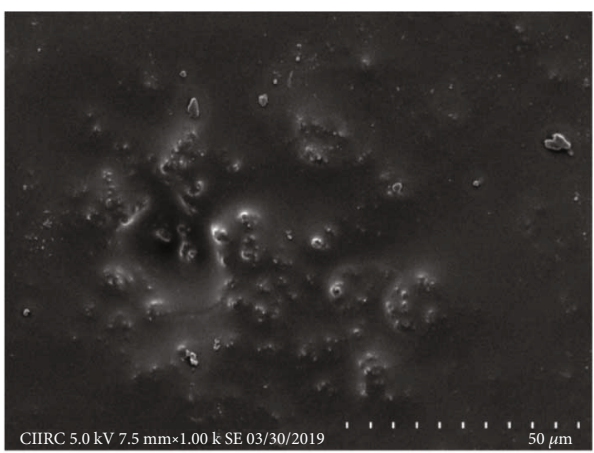

(c)

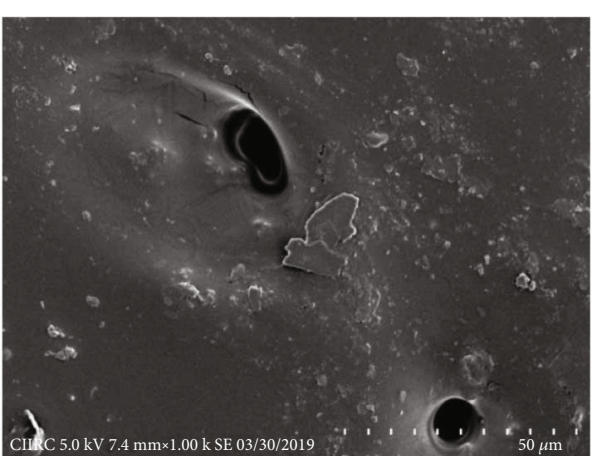

(b)

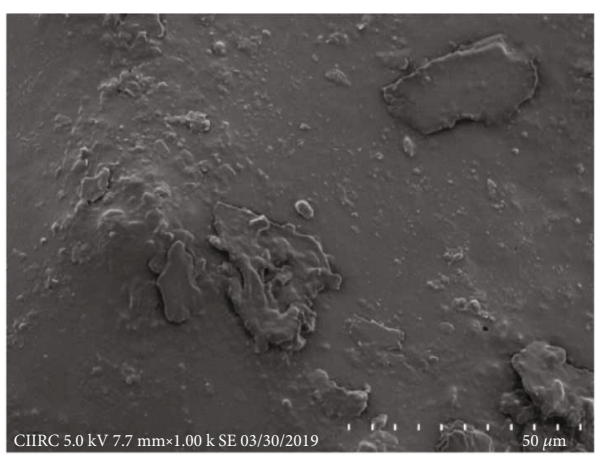

(d)

Figure 11: PFNC-coated steel: (a) blank, (b) 25 ppm, (c) 50 ppm, and (d)100 ppm.

was chosen which is shown in Figures 8(a) and 8(b). Instead of using pure capacitance related to the coating and electrical double layer, a constant phase element is employed to fit the impedance data. When compared to bare and PFNC-coated substrates [18], the charge transfer resistance of PFNCcoated substrates is higher during the initial time of exposure. As illustrated in Figure 9, PFNC-coated substrates exhibited the strongest corrosion resistance even after 48 hours. EIS indicates that PFNC-coated substrate can protect against corrosion even after extended exposure to corrosive conditions.

The Nyquist plots for the MS coated with PFNC and bare substrate in $0.1 \mathrm{M} \mathrm{HCl}$ solutions at $25 \pm 3^{\circ} \mathrm{C}$ are shown in Figure 9. The curve indicates that as the concentration of PFNC increased, the diameter of capacitive loop increased, which represents corrosion inhibition of MS steadily increases.

The formation of partial double-layer capacitance on the mild steel surface is caused by an increase in diameter due to the high frequency of the capacitive loop, which can be accomplished with charging transfer reactions, and this explains the increase in MS resistance with increasing diameter of capacitive loop [10].

3.7.3. Cyclic Voltammetric Studies. Cyclic voltammetry tests were carried out in a one-liter glass cell with an MS counter electrode that ensured uniform current distribution across the specimen surface. The reference electrode was a saturated calomel electrode (SCE) with a potential of $+0.249 \mathrm{~V}$. Just before the cyclic voltammetry test, the MS substrate was removed from the passivation solution.
The CV tests were performed as follows: specimen conditioning at $0.001 \mathrm{~V}$ vs. SCE for $2 \mathrm{~s}$ to clean the surface while avoiding damage to the passive film, $15 \mathrm{~s}$ equilibration at open circuit potential, and two successive voltammetry cycles from 0.0 to $+0.4 \mathrm{~V}$ vs. SCE at $0.1 \mathrm{mV} / \mathrm{s}$ scan rate.

Figure 10(a) depicts a CV curve of MS in $0.1 \mathrm{HCl}$ solution with one high anodic peak potential ranging from 0.0 to $0.4 \mathrm{~V}$ that stabilizes at $0.177 \mathrm{~V}$ after two segments, indicating iron oxidation. Figure 10(b) depicts two unsatisfactory cathodic peak potentials ranging from -1 to $-0.3 \mathrm{~V}$, as well as two peaks that are less evident due to hydrogen development, which stabilizes at -0.467 and. $460 \mathrm{~V}$ after four segments. The magnitude of the peaks grows as the number of segments increases. Figure 10(c) depicts two cathodic peak potentials that span from 0 to $0.4 \mathrm{~V}$ and stabilize at $0.249 \mathrm{~V}, 0.172 \mathrm{~V}$, and $0.249 \mathrm{~V}$ after six segments, respectively. It denotes a decrease in the rate of corrosion [19-21].

3.8. Morphological Studies of PANI, FA, and PFNC. The treatment of the surface of MS substrates with various PFNC compositions significantly improved their corrosion resistance. A considerable corrosion deterioration [22, 23] was observed for substrate in the absence of PFNC (blank) as shown in Figure 11(a). As the substrate was coated with $25 \mathrm{ppm}$ of the PFNC layer, localized corrosion has been noticed due to the tensile stress induced by the thermal treatment (Figure 11(b)). As the concentration level was further enhanced, the rate of corrosion and corresponding deterioration was found decreasing, as seen from the SEM images in Figures 11(c) and 11(d) for $50 \mathrm{ppm}$ and $100 \mathrm{ppm}$ PFNC-coated substrates, respectively. Thus, it can be clearly 
concluded that the PFNS composite can act as a good corrosion inhibitor for mild steel.

\section{Conclusion}

Polyaniline- (PANI-) fly ash (FA) nanocomposite (PFNC) was synthesized using an in situ polymerization method. The synthesized composites were characterized by employing advanced analytical, microscopic, and spectroscopic tools and found that FA stabilized the PANI in emeraldine form. Electrodynamic polarization studies were conducted to study the anticorrosion property of the PFNC-coated substrate. Tafel and cyclic polarization studies confirmed superior corrosion resistance for MS coated with PFNC. In $0.1 \mathrm{M} \mathrm{HCl}$, the findings of potentiodynamic polarization demonstrated that PANI-fly ash nanocomposite is a mixed-type corrosion inhibitor. The anticorrosion capabilities of PFNC increase as concentration rises, as revealed by cyclic voltammetry studies. The FA-doped PANI-coated surfaces produced semicircles with larger diameters, indicating that they are more passivated, according to EIS spectra. All measurements showed that treating the MS surface with PFNC coating can protect the steel against corrosion in $0.1 \mathrm{M} \mathrm{HCl}$ solution to a large extent.

\section{Data Availability}

The experimental data used to support the findings of this study are available from the corresponding author upon request.

\section{Conflicts of Interest}

The authors declare that they have no conflicts of interest.

\section{Authors' Contributions}

The concept and experiments were designed and conducted by Mrs. Karthika Shetty. The original draft of the manuscript was written by Dr. Jayadev. Editing and compiling of the final draft were done by Dr. Kalyan Raj and Dr. H C Ananda Murthy. All authors read and approved the final manuscript.

\section{Acknowledgments}

The authors acknowledge the principal and the management, RNS Institute of Technology Bengaluru for the cooperation and for providing the necessary facilities.

\section{References}

[1] A. N. Ökte and D. Karamanis, "A novel photoresponsive $\mathrm{ZnO}$-flyash nanocomposite for environmental and energy applications," Applied Catalysis B: Environmental, vol. 142143, pp. 538-552, 2013.

[2] A. P. Singh, A. Chandra, and S. K. Dhawan, "Conduction mechanism in Polyaniline-flyash composite material for shielding against electromagnetic radiation in X-band \& $\mathrm{Ku}$ band," AIP Advances, vol. 1, no. 2, article 022147, 2011.
[3] J. C. Jeyageetha, V. Sankaragomathi, M. Bharathi, R. Muthumari, and P. Siji Priya, "Utilization of fly ash: synthesis, spectral, thermal studies of PFNC matrix composites," International Journal of Recent Scientific Research, vol. 7, no. 11, pp. 14466-14473, 2016.

[4] S. C. Raghavendra, S. Khasim, M. V. N. Revanasiddappa, M. V. N. Ambika Prasad, and A. B. Kulkarni, "Synthesis, characterization and low frequency a.c. conduction of polyaniline/fly ash composites," Bulletin of Materials Science, vol. 26, no. 7, pp. 733-739, 2003.

[5] M. M. Popović and B. N. Grgur, "Electrochemical synthesis and corrosion behavior of thin polyaniline-benzoate film on mild steel," Synthetic Metals, vol. 161, pp. 1313-1318, 2011.

[6] D. Dahegaonkar, S. B. Kondawar, and V. A. Thabhane, "Electrical conductivity of chemically synthesized polyaniline fly ash composites," International Journal of Researches in Biosciences, Agriculture and Technology, vol. II, no. 3, pp. 366-370, 2015.

[7] K. Shetty, K. Raj, and N. Mohan, "Synthesis, characterization and corrosion studies of polyanailine(PANI)/ceriem dioxide $\left(\mathrm{CeO}_{2}\right)$ nano composite," Materials Today: Proceedings, vol. 27, pp. 2214-7853, 2020.

[8] K. Shetty and K. Raj, "Synthesized conducting polyaniline $\mathrm{TiO}_{2}$ based nanocomposite for corrosion control on steel 316," Materials Today Proceedings, vol. 515, 2021.

[9] H. Narayan, H. Alemu, and E. Iwuoha, "Synthesis, characterization and conductivity measurements of polyaniline and polyaniline/fly-ash composites," Physica Status Solidi (a), vol. 203, no. 15, pp. 3665-3672, 2006.

[10] S. Gaballah, N. Shehata, M. Shaaban et al., "Corrosion inhibition of aluminium in hydrochloric acid solution using ceria doped polyvinyl chloride nanofiber," International Journal of Electrochemical Science, vol. 12, pp. 1094-1105, 2017.

[11] R. Anbarasan, S. Sivakumaravel, and C. Gopiganesh, "Synthesis and characterizations of poly(aniline)-natural clay nanocomposites," International Journal of Polymeric Materials, vol. 55, no. 10, pp. 803-814, 2006.

[12] R. Prabhu, K. R. Jeevananda, A. V. Reddy, and A. V. Raghu, "Polyaniline-fly ash nanocomposites synthesized via emulsion polymerization: Physicochemical, thermal and dielectric properties," Materials Science for Energy Technologies, vol. 4, pp. 107-112, 2021.

[13] S. Uthaman, R. P. George, V. Vishwakarma, M. Harilal, and J. Philip, "Enhanced seawater corrosion resistance of reinforcement in nanophase modified fly ash concrete," Construction and Building Materials, vol. 221, pp. 232-243, 2019.

[14] P. Patra, I. Roy, R. Kumar et al., "Characterization of nanocomposites in flyash for possible pesticide application," AIP Conference Proceeding, vol. 1276, p. 144, 2010.

[15] M. Harilal, V. R. Rathish, B. Anandkumar et al., "High performance green concrete (HPGC) with improved strength and chloride ion penetration resistance by synergistic action of fly ash, nanoparticles and corrosion inhibitor," Construction and Building Materials, vol. 198, pp. 299-312, 2019.

[16] K. Guin, M. Bhadu, M. Sinhababu, T. K. Rout, and G. Udayabhanu, "Effect of nano $\mathrm{ZnO}$ containing sol-gel coating on galvanised iron sheet," Pigment \& Resin Technology, vol. 44, no. 4, pp. 239-249, 2015.

[17] J. O. Iroh and W. Su, "Corrosion performance of polypyrrole coating applied to low carbon steel by an electrochemical process," Electrochimica Acta, vol. 46, no. 1, pp. 15-24, 2000. 
[18] S. H. Adsul, T. Siva, S. Sathiyanarayanan, S. H. Sonawane, and R. Subasri, "Self-healing ability of nanoclay-based hybrid solgel coatings on magnesium alloy AZ91D," Surface and Coatings Technology, vol. 309, pp. 609-620, 2017.

[19] P. F. Sanaulla, H. C. Ananda Murthy, and V. Bheemaraju, "Corrosion inhibition of AA6061 and AA6063 alloy in hydrochloric acid media by Schiff base compounds," Journal of the Chilean Chemical Society, vol. 57, no. 4, pp. 1364-1370, 2012.

[20] V. Bheemaraju, P. F. Sanaulla, H. B. Lokesh, and H. C. Ananda Murthy, "Electrochemical investigation of corrosion inhibition of AA6063 alloy in 1M hydrochloric acid using Schiff base compounds," IOSR Journal of Applied Chemistry (IOSR-JAC), vol. 2, no. 5, pp. 37-47, 2012.

[21] H. C. A. Murthy and S. Kumar Singh, "Influence of TiC particulate reinforcement on the corrosion behaviour of $\mathrm{Al} 6061$ metal matrix composites," Advanced Materials Letters, vol. 6, no. 7, pp. 633-640, 2015.

[22] H. C. A. Murthy, V. B. Raju, and C. Shivakumara, "Effect of TiN particulate reinforcement on corrosive behaviour of aluminium 6061 composites in chloride medium," Bulletin of Material Science, vol. 36, no. 6, pp. 1057-1066, 2013.

[23] H. C. Ananda Murthy, "Electroanalytical study on the corrosion behaviour of $\mathrm{TiO}_{2}$ particulate reinforced $\mathrm{Al} 6061$ composites," Material Science Research India, vol. 12, no. 2, pp. 112126, 2015. 